\title{
Possible method for evaluation of virus, bacteria and yeasts infectivity by optical measurements
}

\author{
N.M. Rusinchuk, V.Z. Lozovski, O.A. Shydlovska, N.M. Zholobak \\ Taras Shevchenko National University of Kyiv, Institute of High Technologies, \\ 64, Volodymyrs'ka str., 01601 Kyiv, Ukraine \\ E-mail: n.m.rusinchuk@gmail.com
}

\begin{abstract}
The possible method for evaluation of virus, bacteria and yeasts infectivity based on study of their absorption spectra has been proposed. The decrease in the samples infectivity was caused by adding the $\mathrm{CeO}_{2}$ nanoparticles. Optical absorption of the samples was measured for the wavelength range from 400 to $750 \mathrm{~nm}$. The obtained data detect the specific changes in absorption of the samples, which can be used for development of the new technique of evaluation of virus, bacteria and yeasts infectivity.
\end{abstract}

Keywords: Vesicular stomatitis virus, Lactobacillus delbrueckii, Saccharomyces cerevisiae, absorption spectra, extinction coefficient.

Manuscript received 17.03.16; revised version received 04.07.16; accepted for publication 13.09.16; published online 04.10.16.

\section{Introduction}

Existing methods for evaluation of bacterial and especially viral infectivity by measurement of cytopathic effect of cell cultures are very complicated, and the results of these measurements are rather qualitative than quantitative (e.g., Reed and Munch method) [1]. Interaction between the virus and antiviral agents could be visually studied using atomic force microscopy, which is very expensive and not very informative. Hence, there is a need in a new method for evaluation of infectivity, which would be not very expensive and give quantitative results.

Besides, recently interaction between viruses and bacteria with nanoparticles has become more and more relevant problem in modern science. This problem is studied by the scientists all over the world both experimenters and theoretics. Antiviral and antimicrobial effect was already found in experiments with silver [2], gold [3], silica [4] and other nanoparticles. However, there is no exact information on how the nanoparticles inhibit the infectivity of viruses and bacteria.

It is well known that absorption spectrum displays information on the composition of samples. Such parameters as molecular structure, dimensions of the components of the samples and others influence on the absorption spectrum of the sample. Optical studies are widely applied in biomedical studies [5], e.g., determining the bacteria concentration in the sample by using measurements of its absorption at a certain wavelength. Consequently, it can be supposed that optical measurements can be useful for determination of other parameters of the samples, too. Thus, for example, viral (bacterial) samples with different infectivity may have different absorption spectra. Also, it can be supposed that study of light absorption in the samples could be useful for investigation of the interaction between the particles in them. 


\section{Materials and methods}

For the experiments, we used biological samples of various contents. The samples were irradiated with halogen lamp through the lens, and transmitted light was collected using a photoelectric multiplier. The gained signal was processed with computer program. Absorption was calculated as difference between the light intensity transmitted through the cuvette with the buffer and that transmitted through the cuvette with the sample. All the experiments were repeated for five times during one day with the period in one hour in order to eliminate the possibility of virus, bacteria and yeasts destruction in the samples due to the effect of light during the experiment. All the absorption spectra in a series were statistically equal.

As antiviral and antimicrobial therapy with nanoparticles is very relevant method, the study was performed with viral, microbial and yeasts samples with nanoparticles. The experiments were carried out with Vesicular stomatitis Indiana virus (Rhabdoviridae), Lactobacillus delbrueckii and Saccharomyces cerevisiae samples. The nanoparticles were the $\mathrm{CeO}_{2}$ nanoparticles with diameter of 2 to $3 \mathrm{~nm}$ stabilized with citrate.

Absorption spectra were measured for the samples of pure viruses with different virus concentrations, for the samples of viruses with nanoparticles, for the samples of viruses in one week after their preparation and after one-day illumination with direct sunlight, for the samples of bacteria and yeasts with and without nanoparticles, for the samples of buffer.

It should be mentioned that the virus sample consists of not only viruses, but proteins, glycoproteins and other chemicals being in virus in small concentrations. In order to eliminate the influence of these compounds and of inactive viruses in the sample, the absorption spectra for the samples of viruses in one week after their preparation and after one-day illumination with direct sunlight and heating to $50{ }^{\circ} \mathrm{C}$ for half an hour were measured. The influence of high temperature and direct sunlight leads to the inactivation of virus sample. Also, in order to study the possibility of evaluation of parameters of virus sample by measuring its absorption, the samples of viruses with $\mathrm{CeO}_{2}$ nanoparticles were studied.

As a result of series of experiments, the absorption spectra of the samples with account of absorption inherent to the buffer and growth media in the visible spectral range were obtained.

In order to analyze the absorption spectra, its representation in the form of combination of a finite number of oscillators and the Kramers-Kronig relations were used as in Refs. [6, 7]. We assumed that the dielectric function of the preparation is described by the formula [7]:

$\varepsilon(\omega)=\varepsilon_{\infty}+\sum_{i=1}^{N} \frac{f_{i}-i f_{R, i} E}{E_{i}^{2}-E^{2}-i \gamma_{i} E}$, where $\gamma_{i}, f_{i}$ are the damping and the intensity of the $i$-th oscillator, respectively, $E_{i}$ is the energy of photon with angular frequency $\omega_{i}, \omega_{i}$ are the natural frequency of the $i$-th oscillator, and $f_{R, i}$ is the coefficient that characterizes relaxation of the $i$-th oscillator.

The natural frequency of oscillator, its damping and intensity were defined by the presentation of absorption spectra as a sum of absorption curves of each oscillator. The coefficient that characterizes relaxation of the $i$-th oscillator was chosen in such a manner that by least squares method the difference between experimental and simulated extinction coefficient had the lowest value.

\section{Results and discussions}

The obtained absorption spectra for different samples are presented in Figs. 1 to 5. Virus samples after the action of direct sunlight and high temperature demonstrate negligible absorption in the studied wavelength range. As Vesicular stomatitis virus is very unstable, the virus should inactivate under direct sunlight and high temperature. This can indicate accordance of these absorption lines to infective virus.

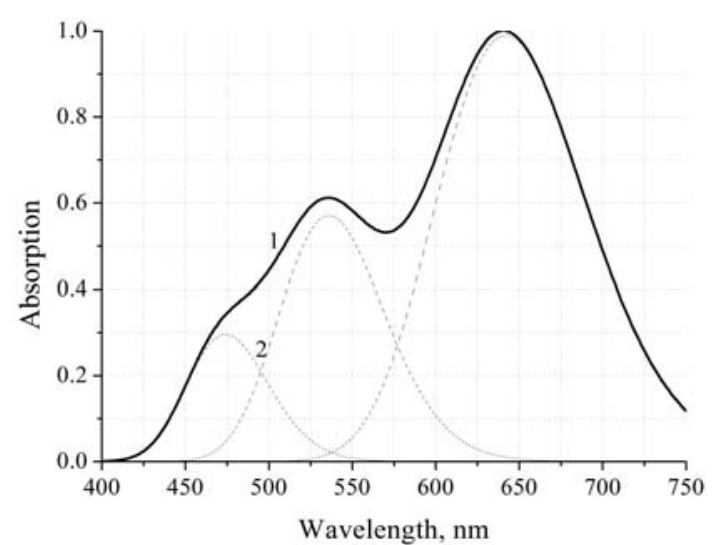

Fig. 1. Absorption spectrum of Vesicular stomatitis sample (curve 1, solid line) and its representation in the form of the sum of oscillators (curve 2, dashed lines).

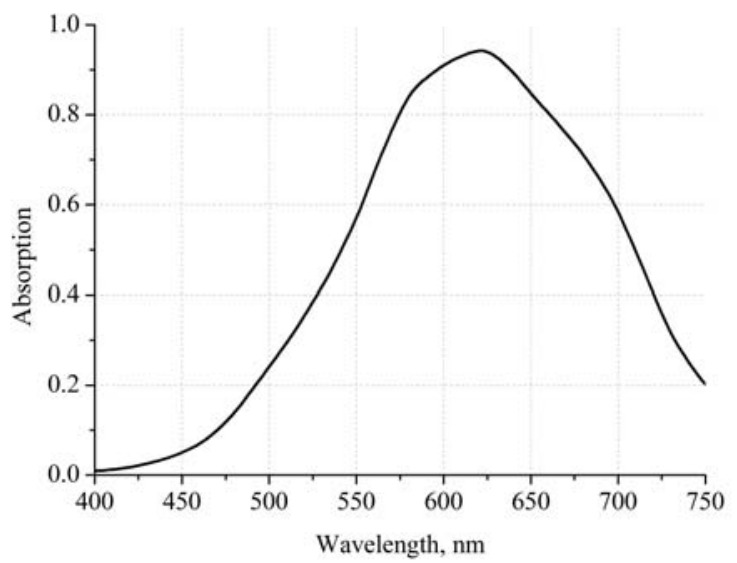

Fig. 2. Absorption spectrum of the $\mathrm{CeO}_{2}$ nanoparticles sample. 


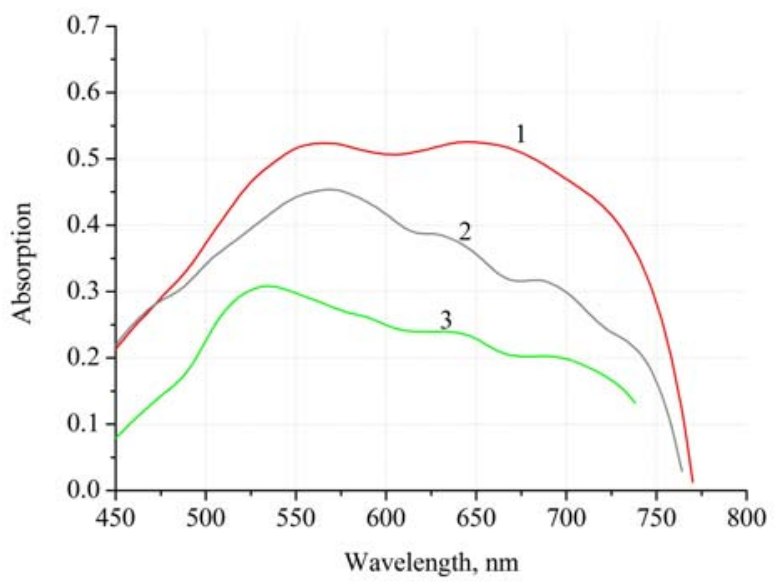

Fig. 3. Absorption spectra of Vesicular stomatitis virus sample with various contents of $\mathrm{CeO}_{2}$ nanoparticles: curve 1 $0.1 \mu \mathrm{Mol} ; 2-1 \mu \mathrm{Mol} ; 3-0.1 \mathrm{mMol}$.

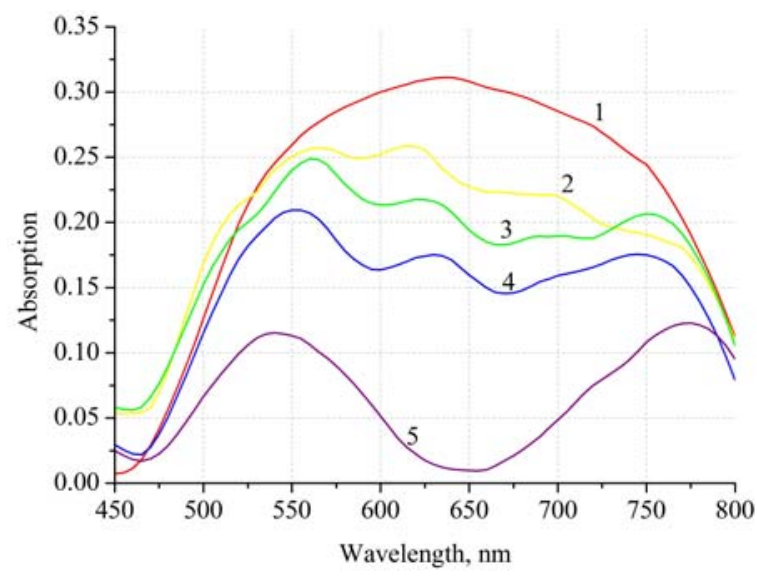

Fig. 4. Absorption spectra of bacteria samples without and with various content of $\mathrm{CeO}_{2}$ nanoparticles: curve 1 - without NP; 2 - $10 \mathrm{nMol} ; 3-1 \mu \mathrm{Mol} ; 4-100 \mu \mathrm{Mol} ; 5-10 \mathrm{mMol}$.

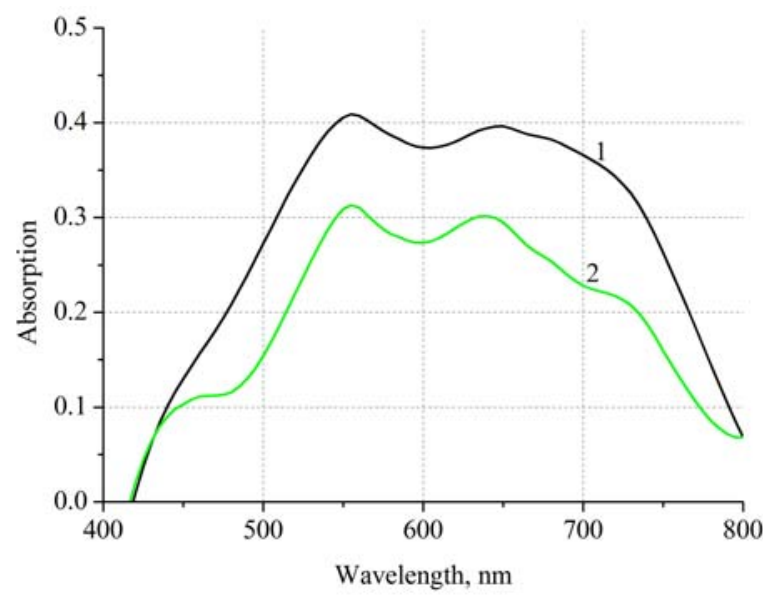

Fig. 5. Absorption spectra of yeasts samples without (curve 1) and with (curve 2) $\mathrm{CeO}_{2}$ nanoparticles.
It was ascertained that addition of the nanoparticles to the sample with viruses, bacteria or yeasts leads to the decrease in the oscillator intensities in varying degrees. Thus, the intensities of the oscillators with the higher frequencies decrease more than those with lower frequencies. The decrease is more considerable when the concentration of nanoparticles is higher.

It is well known that the samples with nanoparticles have antiviral and antimicrobial effect [8-12]. Furthermore, the performed studies of antiviral action of various nanoparticles show that the higher the nanoparticle concentration, the higher their antiviral and antimicrobial effect [8-12]. Thus, the results are in good agreement with the results of biomedical experiments.

Also, it should be noted that absorption of the virus samples after the action of the direct sunlight during one day is negligible. Thus, it can be concluded that adding the nanoparticles to the viruses (bacteria) does not destroy them, but suppresses their infectivity due to interaction between the viruses (bacteria) and nanoparticles (e.g., as in $[9,10])$. Hence, the described method could be useful for evaluation of changes in infectivity due to addition of the nanoparticles, while the shape and the intensity of absorption spectrum of the viral (bacterial) sample can give information on its content and virus or bacterial infectivity.

\section{Conclusions}

The possibility to use optical measurements for studying interaction of viruses, bacteria and yeasts with nanoparticles as well as evaluation of infectivity was shown. The changes in the oscillator intensities correlate with inhibition of infectivity with nanoparticles due to action of the nanoparticles.

Thus, being based on the results of the work, the method for measuring the absorption spectra of virus, bacteria and yeasts samples can be recommended to use for further studies of mechanism of interactions in the samples. Accordance between the changes in light absorption and virus (or bacteria) infectivity should be studied in more detail to develop a new possible method for evaluation of infectivity.

\section{Acknowledgements}

We are grateful to M.Ya. Spivak and V.S. Lysenko for fruitful discussions and support of the work performance.

\section{References}

1. L.J. Reed, H. Muench, A simple method of estimating fifty percent endpoints // Amer. J. Hygiene, 27, p. 493-497 (1938).

2. L. Lu, S.R. Wai-Yin, R. Chen et al., Silver nanoparticles inhibit hepatitis B virus replication // Antiviral Therapy, 13, p. 253-262 (2008). 
3. D. Baram-Pinto, S. Shukla, A. Gedanken, and R. Sarid, Inhibition of HSV-1 attachment, entry, and cell-to-cell spread by functionalized multivalent gold nanoparticles // Small, 6, p. 1044-1050 (2010).

4. D. Botequim, J. Maia, M.M.F. Lino et al., Nanoparticles and surfaces presenting antifungal, antibacterial and antiviral properties // Langmuir, 28, p. 7646-7656 (2012).

5. V.Y. Maleev, Methods of Biophysical Studies. V.N. Karazin Kharkiv National University, 2014 (in Russian).

6. E.G. Bortchagovsky, U.C. Fischer, Method for determination of the dielectric function of a thin absorbing film on variable substrates from transmission spectra // Appl. Opt. 42, p. 6915-6918 (2003).

7. V.B. Shirokov, Y.I. Golovko, V.M. Muhortov, Optical parameters of $\mathrm{BiFeO}_{3}$ epitaxial thin film // J. Techn. Phys. 84, p. 104-108 (2014).

8. N.M. Zholobak, Z.M. Olevinskaia, N.Ya. Spivak et al., Antiviral effect of cerium dioxide nanoparticles stabilized by low-molecular polyacrylic acid // Mikrobiolohichnyi Zhurnal, 72(3), p. $42-47$ (2010), in Ukrainian.

9. V. Lozovski, V. Lysenko, V. Piatnytsia, O. Scherbakov, N. Zholobak, and M. Spivak, Physical point of view for antiviral effect caused by the interaction between the viruses and nanoparticles// J. Bionanosci. 6, p. 109-112 (2012).

10. V. Lysenko, V. Lozovski, M. Spivak, Nanophysics and antiviral therapy // Ukr. J. Phys. 58, p. 77-90 (2013).

11. S. Gaikwad, A. Ingle, A. Gade et al., Antiviral Activity of Mycosynthesized Silver Nanoparticles against Herpes Simplex Virus and Human Parainfluenza Virus Type 3 // Intern. J. Nanomedicine, 8(1), p. 4303-4314 (2013).

12. Y. Mori, T. Ono, Y. Miyahira, V.Q. Nguyen, T. Matsui, M. Ishihara, Antiviral Activity of Silver Nanoparticle/Chitosan Composites against H1N1 Influenza A Virus // Nanoscale Res. Lett. 8, p. 93 (2013). 